\title{
Les manuscrits brûlent (matérialité de l'écrit dans les poèmes épistolaires de Iosif Brodskij)
}

Yan Walther

\section{(2) OpenEdition}

1 Journals

Édition électronique

URL : http://journals.openedition.org/edl/322

DOI : $10.4000 /$ edl.322

ISSN : 2296-5084

Éditeur

Université de Lausanne

\section{Édition imprimée}

Date de publication : 15 décembre 2009

Pagination : 167-178

ISBN : 978-2-940331-21-5

ISSN : 0014-2026

Référence électronique

Yan Walther, «Les manuscrits brûlent (matérialité de l'écrit dans les poèmes épistolaires de losif Brodskij) », Études de lettres [En ligne], 4 | 2009, mis en ligne le 15 décembre 2012, consulté le 18 décembre 2020. URL : http://journals.openedition.org/edl/322 ; DOI : https://doi.org/10.4000/edl.322 


\section{LES MANUSCRITS BRÛLENT \\ (MATÉRIALITÉ DE L'ÉCRIT DANS LES POÈMES ÉPISTOLAIRES DE IOSIF BRODSKIJ)}

En réactualisant le lien devenu conventionnel entre le poème épistolaire et la lettre en tant qu'objet, I. Brodskij met en relief la matérialité du texte, et par là, métaphoriquement, sa fragilité. Cette idée lui permet de symboliser trois thèmes importants de son œuvre: le danger qui menace la littérature; la fragilité de toute communication humaine; la culture vue comme «fil d'Ariane» de la civilisation.

\section{Le genre épistolaire chez Brodskij}

Le genre épistolaire occupe une place importante dans l'œuvre poétique de Iosif Brodskij. De ses débuts littéraires (par exemple «Dans une lettre au sud» ["V pis'me na jug»] [I, 84; 1961] ${ }^{1}$ ) jusqu’à la fin de son œuvre, il a composé avec régularité plus d'une trentaine de poèmes en forme de lettre, parmi lesquels des textes aussi cruciaux que «Lettre dans une bouteille» [ [Pis'mo v butylke»] (I, 362; 1964) et «Lettres à un ami romain" [«Pis'ma rimskomu drugu»] (II, 284;03. 1972).

On peut y voir tout d'abord l'influence de facteurs historiques et biographiques : la correspondance écrite était un phénomène non seulement

I. Cf. I.A. Brodskij, Sočinenija v 5-i tomax. Toutes les références renvoient à cette édition. Le chiffre romain indique le tome, le chiffre arabe la page. La date de composition est indiquée finalement après un point-virgule lors de la première mention d'un poème. Les parties des poèmes numérotées par l'auteur seront désignées par le signe $\$$. Les paragraphes et les vers seront indiqués après une citation. 
très répandu, mais également vital en URSS. Le journaliste et historien L. Parfenov l'explique très bien dans sa série documentaire télévisée L'autre jour: Notre ère [Namedni-Naša èra] :

Une très grande partie de ce très grand pays vivait par correspondance. Le téléphone n'était pas un phénomène de masse. Le téléphone interurbain encore moins. Et les parents qui vivaient dans des villes différentes ne gardaient contact durant des années qu'en s'écrivant des lettres; et avant les fêtes, on envoyait un tas de cartes de vœux ${ }^{2}$.

D'autre part, au cours d'expéditions géologiques, de son exil au village de Norenskaja de mars 1964 à septembre 1965 et finalement de son exil définitif aux Etats-Unis dès juin 1972, Brodskij a lui-même fait l'expérience de la séparation, de la distance d'avec ses proches de façon tout à fait authentique, et il parait évident que la correspondance qui le maintenait en contact avec eux devait avoir une importance toute particulière pour lui.

De fait, l'un des traits originaux de plusieurs de ses poèmes épistolaires, qui démontre d'ailleurs la recherche d'un effet concret et réaliste, est l'allusion à la matérialité de l'écrit et aux détails pratiques de l'expédition comme parties intégrantes du phénomène de la correspondance, voire du genre épistolaire lui-même. Cela implique que de tels poèmes se donnent non pas comme une parole pure, désincarnée, mais bien comme un texte écrit sur du papier et envoyé dans une enveloppe (ou dans une bouteille à la mer!), qui parcourra physiquement la distance qui sépare l'auteur du destinataire.

\section{Fragilité de l'écrit}

Cet effet est utilisé de façon frappante dans "Lettre dans une bouteille", dont plusieurs passages ont été effacés par l'eau («razmyto»). La dé(con) struction qui en résulte permet ici de mettre le texte en valeur par sa propre absence ${ }^{3}$ et, en rompant la continuité narrative supposée de

2. L.G. Parfenov, Namedni. Notre traduction, comme toutes celles de ce travail. Remarque: l'apparition des codes postaux en URSS date de 1971.

3. Nous retrouvons cet effet dans d'autres poèmes, par exemple: «Intermezzo de juillet» ["Ijul’skoe intermecco»] (I, 84, \$3; 06. 1961), «Strophes» («En guise d'adieu: pas un son») [«Strofy (Na proščanie - ni zvuka)»] (II, 94; 1968), etc. 
la lettre, de renforcer le caractère dramatique de la disparition de son auteur. Plus généralement, ce procédé met en valeur la fragilité de la lettre comme support, et donc du texte lui-même, qui devient dès lors une métaphore de la vie de son auteur. La récurrence de cette idée dans d'autres poèmes (qui, sans être véritablement des lettres, sont très proches du genre épistolaire) témoigne de son importance pour Brodskij. Ainsi, dans "Explorateur polaire» ["Poljarnyj issledovatel” "] (II, 443; 22. 07. 1978), le narrateur n’a plus de papier pour écrire:

Vse sobaki s"edeny. V dnevnike ne ostalos' čistoj stranicy. I biser slov pokryvaet foto suprugi, $\mathrm{k}$ ee ščeke mušku daty somnitel'noj prikolov ${ }^{4}$.

Et l'impossibilité matérielle d'écrire signifie clairement sa mort. Inversement, mais selon la même logique, dans "Mon second Noël au bord...» ["Vtoroe Roždestvo na beregu...»] (II, 264; 1971), le lien entre la vie et le texte, qui dépend de son support matériel, est direct:

I ja ne mogu skazat', čto ne mogu

žit' bez tebja - poskol'ku ja živu.

Kak vidno iz bumagi ${ }^{5}$.

En même temps, l'écriture d'une lettre est un symbole de la littérature, ce qui montre bien le lien étroit qui unit vie et littérature pour Brodskij. En effet, dans "Un nouveau Jules Verne» ["Novyj Žjul' Vern»] (II, 387; 1976), la remarque: «Čudo, čto pis'mennye prinadležnosti i tvoja fotokartočka uceleli» [C'est un miracle que mon écritoire et ta photographie ${ }^{6}$ aient survécu] $(\$ 9$, v. 2), outre l'amusante touche de réalisme

4. "J'ai mangé le dernier chien. Dans mon journal / plus une page vierge. Des perles de mots / recouvrent la photo de mon épouse, collant sur sa joue / la mouche d'une date douteuse» (v. 1-4).

5. «Et je ne peux pas dire que je ne peux pas / vivre sans toi: puisque je vis. I Comme tu le vois au papier» (v. 4-6).

6. Un détail intéressant: dans «Un nouveau Jules Verne» comme dans «Explorateur polaire", l'acte d'écriture est associé à la photographie de la fiancée. Celle-ci peut être vue comme une métaphore de la mémoire et de l'amour dont nous comprenons ainsi qu'ils sont des conditions essentielles à la littérature pour Brodskij. Les vers suivants sont tirés de «Ma chère, je suis sorti tard ce soir de ma maison..." [ "Dorogaja, ja vyšel segodnja iz domu pozdno večerom...»] (III, 184): "Povezlo i tebe: gde ešče, krome razve čto fotografii, / ty prebudeš vsegda bez morščin, moloda, vesela, glumliva? / Ibo 
qu'elle ajoute à cette situation parfaitement fantastique ${ }^{7}$ implique clairement la dépendance de l'écriture à certaines conditions matérielles: sans écritoire, pas de lettre. Métaphoriquement, nous y lisons la fragilité de la littérature et sa dépendance aux contingences extérieures (matérielles, politiques, etc.) dans le monde post-apocalyptique de Brodskij ${ }^{8}$.

En somme, comme dans "Lettre dans une bouteille», la lettre, symbole de la littérature, est un messager miraculeux, capable de surmonter des obstacles aussi dangereux qu'improbables (le système digestif d'un poulpe géant, par exemple ${ }^{9}$ ), sans pourtant n'être jamais assurée de parvenir à bon port: la correspondance peut s'interrompre à chaque instant, comme dans "Un nouveau Jules Vernes " ${ }^{10}$. De même, dans un poème comme «Exhortation» [«Nazidanie»] (III, 130; 1987):

V pis'max iz ètix mest ne soobščaj o tom, s čem stolknulsja v puti. No, šelestja listom, povestvuj o sebe, o čustvax i proč. - pis’mo mogut perexvatit' ${ }^{\prime 1}$,

Brodskij insiste sur le danger qui pèse sur toute correspondance, sur le fait que, comme la littérature, elle n'est pas donnée d'avance, elle n'est jamais à l'abri. C'est pour cette raison que l'auteur d'une lettre ne sait jamais si son destinataire la recevra, idée que nous trouvons chez Brodskij dès sa "Lettre à A. D.» ["Pis'mo k A. D.»] (I, 160; 27. 01. 1962):

vremja, stolknuvšis' s pamjat'ju, uznaet o svoem bespravii» [Et toi aussi, tu as de la chance: où encore, ailleurs que sur les photographies, / seras-tu toujours sans rides, jeune, joyeuse, railleuse? / Car le temps, s'étant heurté à la mémoire, se rend compte qu'il n'a pas de droits] (v. 17-19). - Y. W.

7. "Dorogaja Blanš, pišu tebe sidja vnutri gigantskogo os'minoga» [Chère Blanche, je t'écris assis dans un gigantesque poulpe] (\$IX, v. 1).

8. Monde de l'après Seconde guerre mondiale, du communisme, monde qui rappelle aussi celui de «La Terre vaine» ["The Waste Land»] de T.S. Eliot, l'un des maîtres de Brodskij...

9. «Postarajus' issledovat' sistemu piščevaren'ja. / Ėto - edinstvennyj put'k svobode" [Je vais essayer d'étudier le système digestif. / C'est le seul chemin vers la liberté], cf. "Un nouveau Jules Verne» (II, 391, \$IX, v. 7-8).

IO. "(Zdes' obryvajutsja pis'ma k Blanš Delarju ot lejtenanta Benca)» [Ici s'interrompent les lettres à Blanche Delarue du lieutenant Benz] (II, 392, après le \$9).

II. «Dans tes lettres de ces contrées, ne raconte pas / ce que tu as vu en chemin. Plutôt, faisant bruire la feuille, / parle de toi, de tes sentiments, etc.: la lettre / peut être interceptée» (III, 132, \$10, v. 1-4). 
Vse ravno ty ne slyšišs, vse ravno ne uslyšiš̌ ni slova, vse ravno ja pišu, no kak stranno pisat' tebe snova, no kak stranno opjat' soveršat' povtoren'e proščan'ja, dobryj večer. Kak stranno vtorgat'sja v molčan'e ${ }^{12}$.

Dans «Lettre au Général Z.» [ Pis'mo Generalu Z.»] (II, 85; 1968), l’officier démissionnaire n'est pas sûr que ses paroles, sa lettre parviendront au général :

General! Esli vse-taki vy menja

slyšite, značit, pustynja prjačet

nekij oazis v sebe, manja

vsadnika ètim; a vsadnik, značit,

ja; ja prišporivaju konja;

kon', general, nukuda ne skačet ${ }^{13}$.

En résumé, le recours à la matérialité de l'écrit permet à Brodskij de souligner sa fragilité (non seulement les manuscrits brûlent, mais ils peuvent être détrempés, interceptés, digérés...!), et avec elle celle de la littérature qu'il symbolise. D'autre part, comme le montrent les trois derniers exemples, la mise en avant de la fragilité de la correspondance comme moyen de communication permet également de faire allusion au fait que le poème est avant tout écrit parce qu'il doit être écrit, pour soi, indépendamment de sa réception par un hypothétique lecteur.

I2. «De toute façon tu n'entends pas, de toute façon tu n'entendras pas un mot, / de toute façon je t'écris, mais comme c'est étrange de t'écrire à nouveau, / mais comme c'est étrange d'accomplir encore la répétition d'un adieu, / bonsoir. Comme c'est étrange de faire irruption dans le silence» (v. 1-4).

I3. "Général! Si malgré tout vous m' / entendez, cela veut dire que le désert recèle / en lui quelque oasis, attirant / grâce à elle le cavalier; et ce cavalier, en somme, c'est / moi; j'éperonne mon cheval; / le cheval, général, ne galope nulle part» (II, 89, \$6, v. 7-12). 


\section{Raffinement et barbarie}

Il y a également une allusion tout à fait directe à la matérialité du texte dans les «Lettres de la dynastie Ming» [«Pis'ma dinastii Min’»] (II, 426; 1977) : elles sont écrites à l'encre de Chine ${ }^{14}$ sur du papier de riz fin donné par l'impératrice à "Canard Sauvage». L'écriture est ainsi associée au raffinement d'une civilisation. Or il y a un rapport très ambigu chez Brodskij entre la barbarie et la civilisation. En effet, tantôt c'est la barbarie qui entraîne le chaos et la mort, auxquels s'oppose l'écriture comme symbole d'une civilisation porteuse de culture, tantôt c'est la civilisation, en tant qu'elle opprime l'individu (impérialisme, totalitarisme, extrémisme religieux...), qui est malfaisante, et c'est à elle que s'oppose alors une littérature qui incarne et défend les valeurs de l'individu.

Ce paradoxe est manifeste dans le poème précité, puisque le raffinement chinois de l'écriture y est aussi celui de la violence de l'empire porteur de cette civilisation: le Bogdyxan "fait passer» ("zapivaet»!) ses pilules contre le mal de tête avec le sang d'un tailleur "fautif" ("proštrafivšijsja»). L'ambivalence est d'ailleurs poussée plus loin par la remarque qui conclut la première lettre: «Počemu-to vokrug vse bol'še bumagi i vse men'še risa" [Je ne sais pourquoi, il y a de plus en plus de papier et de moins en moins de riz par ici], où nous voyons une allusion au fait que le riz, nourriture essentielle, est utilisé à faire du papier, objet de luxe, alors même que les pauvres gens ne mangent probablement pas à leur faim.

Preuve de l'importance de cette thématique pour Brodskij, nous retrouvons la même ambiguïté dans "Guèrnavaca» [Guernavaka»], premier poème du cycle «Divertissement mexicain» ["Meksikanskij divertisment »] (II, 366; 1975) - M. ${ }^{15}$ incarne la civilisation européenne, avec son raffinement qui n'est pas toujours de circonstance:

I4. Remarquons au passage ce qui est sans doute une allusion biographique: le poème datant de 1977, les treize ans mentionnés dans le vers 1 renvoient à 1964, année où Marina Basmanova trompa Brodskij avec Bobyšev; d'autre part, selon le poème «Ma chère, je suis sorti tard ce soir...», dédié à M. B., l'encre de Chine ("tuš"») lui est associée: «ty [...] risovala tuš ju v bloknote...» [tu (...) dessinais à l'encre de Chine dans un bloc-notes...] (v. 6).

I5. Maximilien d'Autriche (le frère de François Joseph I d'Autriche), envoyé au Mexique par la France comme empereur (1864-1867). Il fut fusillé sur ordre de Benito Juarez. 
On vvel xrustal', šampan'skoe, baly.

Takie vešči skrašivajut byt.

Zatem respublikanskaja pexota

M. rasstreljala ${ }^{16}$

ainsi qu'avec son immoralité, qui se devine à la grossièreté de l'allusion à sa "mulâtresse» (que l'on imagine facilement en esclave sexuelle, tribut au colonialisme). La civilisation européenne n'est donc en rien idéalisée ${ }^{17}$. Pourtant, la pratique de la correspondance symbolise ici aussi la survie de la culture en milieu hostile, et lorsque M. demande de façon touchante à son frère: "Pošlite al'manaxov i poèm" [Envoyez-moi des almanachs et des poèmes] $(\$ 2, \text { v. } 21)^{18}$, cela renvoie à la civilisation de la culture.

Ainsi, preuve de la richesse évocatoire que Brodskij tire de ce procédé, les allusions à la matérialité de l'écrit et à la correspondance épistolaire dans sa pratique (pratique, remarquons-le, qui est toujours liée à un danger) permettent également de souligner l'importance de la lettre comme fil d'Ariane de la civilisation. Et comme nous l'avons vu, c'est d'autant plus significatif que, pour Brodskij, la lettre symbolise la littérature.

I6. "Il introduisit la vaisselle en cristal, le champagne, les bals. / Le genre de choses qui embellissent la vie de tous les jours. / Puis l'infanterie républicaine / fusilla M.» (v. 9-12).

I7. Malgré tout, nous apprenons plus loin dans le cycle que pour Brodskij, à choisir: «Vse-taki lučše sifilis, lučše žerla / edinorogov Kortesa, čem èta žertva. / Eželi vam glaza suždeno skormit' voronam, / lučše esli ubijca - ubijca, a ne astronom. / Voobšče bez ispancev vrjad li by im slučilos / tolkom uznat', čto voobšče slučilos' " [Malgré tout mieux valent la syphilis, mieux les bouches / des obusiers de Cortès, que cette victime [c'est-à-dire, d'un sacrifice - Y.W.]./ S'il vous échoit de nourrir les corbeaux de vos yeux, / autant que votre assassin soit un assassin, et pas un astronome. / En fait, sans les Espagnols, il ne leur aurait jamais été donné / de comprendre, ce qui s'est vraiment passé], cf. «A Eugène» [ K Evgeniju»], sixième poème du cycle «Divertissement mexicain» (II, 374, v. 19-24).

I8. Comparer avec "Lettres à un ami romain» (II, 284; 03. 1972), \$2, v. 1: «Posylaju tebe, Postum, èti knigi» [Je t'envoie ces livres, Postume]. 


\section{Matérialité des mots et des caractères}

Finalement, une dernière preuve de l'importance accordée à la matérialité de l'écrit nous est fournie par les nombreuses allusions et métaphores qui, chez Brodskij, renvoient aux caractères et aux mots eux-mêmes. Par exemple, dans "Lettre à un archéologue» [ Letter to an archeologist»] (III, 384; 1983), le texte prétend être écrit dans une langue antique qu'on peut déchiffrer mais qu'on ne saurait pas prononcer: "Don't reconstruct those vowels, / consonants, and so forth" [ $\mathrm{Ne}$ reconstruis pas ces voyelles, / ces consonnes, et catera] (v. 13). Il fait évidemment allusion aux manuscrits et aux tablettes anciennes, et donc à une écriture on ne peut plus matérielle. De ce point de vue, il est tout à fait remarquable que, contrairement à la poésie lyrique qui s'identifie volontiers au chant, ou du moins à la parole, la lettre, chez Brodskij, se veut un texte muet, inerte en quelque sorte, conséquence de sa matérialisation. Il n'est dès lors pas étonnant que, comme dans l'exemple précédent, les métaphores renvoient à la vue et au toucher, et jamais à l'ouïe.

Par ailleurs, dans certaines œuvres de Brodskij, le poème se place du point de vue du lecteur et renvoie donc à sa propre lecture, à son déchiffrage, en soulignant la matérialité des caractères et des mots eux-mêmes. Par exemple, il en est ainsi dans "Tu me reconnaîtras à mon écriture..." ["Ty uznaeš menja po počerku...»] (III, 154; 1987), dans "Au siècle suivant» " $V$ sledujuščij vek»] (IV, 24; 1994) ${ }^{19}$ ou encore dans «Le crépuscule. La neige. Le silence. Tout est très...» [ «Sumerki. Sneg. Tišina. Ves'ma...»] (II, $21 ; 1966)^{20}$.

Ce procédé culmine dans des métaphores du type: «Sad gust, kak tesno nabrannoe $\check{Z}$." [Le jardin est touffu comme un $F$ calligraphié;

I9. "Ty pročteš” èti bukvy, ostavšiesja ot pera..." [Tu liras ces caractères laissés par ma plume...] (v. 2).

20. «Èti stroki v tvoi persty / popav..." [Ces lignes, entre tes doigts / une fois parvenues...] (v. 11-12). Plus généralement, dans ce poème, c'est toute l'allégorie entre la lecture d'une lettre et la succession des saisons qui est construite sur des métaphores qui matérialisent l'écriture. Par exemple: «Pestrotu ijulja, zelen' vesny / osen' prevraščaet v černye stroki, / i zima čitaet ee upreki / i začityvaet do belizny" [La chamarrure de juillet, la verdure du printemps, / l'automne les transforme en lignes noires, / et l'hiver lit ses reproches, / et les lit et les relit jusqu'à la blancheur] (v. 21-24). 
traduction approximative, littéralement comme un $\check{Z}$ écrit serré] ${ }^{21}$, où le caractère lui-même sert de terme de comparaison ${ }^{22}$. Le grand nombre de métaphores similaires dans l'œuvre de Brodskij, y compris dans les poèmes non épistolaires, démontre bien l'importance du thème de la matérialité de l'écrit.

\section{Conclusion: Le danger}

Par cette courte étude, nous avons voulu montrer que, pour Brodskij, le poème épistolaire n'est pas seulement un genre littéraire conventionnel, mais qu'il renvoie à la lettre comme objet, dans son existence matérielle. Dès lors, les allusions au support du texte, aux processus de l'écriture, de l'expédition, etc. sont autant de moyens d'instaurer une distanciation d'avec le texte, d'amener le lecteur à considérer les mots non seulement comme des signifiants, mais comme des signes.

2I. "Guèrnavaca», $\$ 1$, v. 4. La lettre russe qui correspond au son [ž] ressemble à un $X$ avec une barre verticale au milieu.

22. Cf. également: «La Tamise à Chelsea» ["Temza v Čelsi»] (II, 350; 1974), \$IV, v. 5: "“Kak ty žil v èti gody?” - "Kak bukva g v ogo” " [" Comment vivais-tu alors?” - "Comme la lettre $s$ dans oso" "; traduction approximative] ; "Décembre à Florence» [«Dekabr’ vo Florencii»] (II, 383; 1976) \$VIII, v. 6-8: «Policejskij na perekrestke / mašet rukami, kak bukva $\check{z}$, ni vniz, ni / vverx» [Le policier au carrefour / agite les bras, comme la lettre $X$, sans dessus ni / dessous; traduction approximative, cf. la note précédente]; «Lettres de la dynastie Ming»: «...čelovek urodliv i strašen, kak ieroglif, / kak ljubye nerazborčivye pis'mena» [... l'homme est affreux et laid, comme un hiéroglyphe, / comme tous les autres alphabets indéchiffrables] (\$II, v. 11-12); «Strophes» ("Pareillement à un verre...») ["Strofy (Napodob’e stakana...»] (II, 455; 1978): "Kak tridcat' tret'ja bukva, / ja pjačus' vsju žizn' vpered " [Comme la trente troisième lettre, / toute ma vie j'avance à reculons] (\$X, v. 3-4 ) - il s'agit de la lettre russe qui correspond au son [ja] et qui a la forme d'un $R$ tourné à gauche; "Dédié à une chaise " ["Posvjaščaetsja stulu»] (III, 145; 1987) : «Na mjagkij v profil’ smaxivaja znak / i "vosem" ", no kvadratnoe, v anfas, / stoit on v centre komnaty..." [Rappelant de profil le signe mou / et "huit", mais carré, de face, / elle (la chaise) se tient au centre de la pièce...] (\$3, v. 1-3); «Centaures IV» [«Kentavry IV»] (III, 166; 1988) : «Bez povodka ot vladel'cev ne otlitčit' sobak, / v knige vtoraja bukva vygljadit slepkom s pervoj...» [Sans laisse, on ne distinguerait pas les chiens des maîtres, / dans le livre, la deuxième lettre semble identique à la première...] (v. 9-10); «Nouvelle vie» [Novaja žizn”»] (III, 167 ; 1988): "...stul'ja kak bukva $b$ / ili kak mjagkij znak..." [...des chaises comme la lettre $b$ / ou comme le signe mou...] (v. 9-10), etc. 
Surtout, en évoquant la matérialité du texte et la pratique concrète de la correspondance, Brodskij tisse une véritable allégorie qui met en parallèle l'écriture de la lettre avec la littérature, allégorie où la fragilité du texte en tant qu'objet fait écho aux dangers (symbolisés par les naufrages, la guerre...) qui menacent la littérature.

Finalement, la fragilité du texte est encore un moyen de mettre en relief la frontière qui sépare tout auteur de son lecteur, qu'il s'agisse d'un poème ou d'une simple lettre, et donc la question de la communication entre les êtres. Le fait que Brodskij ait interrogé dans toute son œuvre la possibilité même de la communication en lien avec la mission de la poésie explique dès lors très bien la place d'honneur qu'il a accordée au genre épistolaire.

Yan Walther

Université de Lausanne 


\section{BIBLIOGRAPHIE}

Brodskij, Iosif Aleksandrovič, Sočinenija v 5-i tomax, Sankt-Peterburg, Puškinskij fond, 1992.

Parfenov, Leonid Gennad'evič, Namedni. Naša èra. 1961-2003; 1971. Telekompanija «NTV», 2004 (matériel audio-visuel). 
